Article

\title{
CRISPR/Cas9-Mediated Gene Replacement in the Fungal Keratitis Pathogen Fusarium solani var. petroliphilum
}

\author{
Jorge D. Lightfoot ${ }^{1, *}$ and Kevin K. Fuller ${ }^{1,2, *}$ \\ 1 Department of Microbiology and Immunology, University of Oklahoma Health Sciences Center, \\ Oklahoma City, OK 73104, USA \\ 2 Department of Ophthalmology, University of Oklahoma Health Sciences Center, \\ Oklahoma City, OK 73104, USA \\ * Correspondence: jorge-lightfoot@ouhsc.edu (J.D.L.); kevin-fuller@ouhsc.edu (K.K.F.); \\ Tel.: +405-271-8000 (J.D.L.); +405-271-3695 (K.K.F.)
}

Received: 13 September 2019; Accepted: 12 October 2019; Published: 16 October 2019

\begin{abstract}
Fungal keratitis (FK) is a site-threatening infection of the cornea associated with ocular trauma and contact lens wear. Members of the Fusarium solani species complex (FSSC) are predominant agents of FK worldwide, but genes that support their corneal virulence are poorly understood. As a means to bolster genetic analysis in FSSC pathogens, we sought to employ a CRISPR/Cas9 system in an FK isolate identified as Fusarium petroliphilum. Briefly, this approach involves the introduction of two components into fungal protoplasts: (1) A purified Cas9 protein complexed with guide RNAs that will direct the ribonuclease to cut on either side of the gene of interest, and (2) a "repair template" comprised of a hygromycin resistance cassette flanked by 40 bp of homology outside of the Cas9 cuts. In this way, Cas9-induced double strand breaks should potentiate double homologous replacement of the repair template at the desired locus. We targeted a putative ura3 ortholog since its deletion would result in an easily discernable uracil auxotrophy. Indeed, $10 \%$ of hygromycin-resistant transformants displayed the auxotrophic phenotype, all of which harbored the expected ura3 gene deletion. By contrast, none of the transformants from the repair template control (i.e., no Cas9) displayed the auxotrophic phenotype, indicating that Cas 9 cutting was indeed required to promote homologous integration. Taken together, these data demonstrate that the in vitro Cas 9 system is an easy and efficient approach for reverse genetics in FSSC organisms, including clinical isolates, which should enhance virulence research in these important but understudied ocular pathogens.
\end{abstract}

Keywords: Fusarium solani; Fusarium solani species complex; Fusarium petroliphilum; CRISPR/Cas9; genome editing; ura3 gene; 5-FOA

\section{Introduction}

The transparency of the cornea-due to its avascularity, paucity of resident cells, and careful assembly of collagen fibrils-allows for the entry of light into the eye for proper vision [1]. Following damage to the corneal epithelium, however, microbes can gain access to the underlying stroma and induce an inflammatory response (keratitis) that results in pain, photophobia and acute vision loss. Various microbes can cause keratitis (e.g., bacteria, amoeba and viruses), but fungi are associated with the highest rates of complications and poorest visual outcomes [2]. Approximately 20-40\% of fungal keratitis (FK) cases do not resolve with antifungal intervention, resulting in the need for one or more corneal transplants [3].

Classical risk factors for the development of FK include tropical climate and ocular trauma [4]. In south India, for example, up to half of all microbial keratitis cases are fungal and such infections 
are common among agricultural laborers who develop corneal abrasions from vegetative debris [5]. A fungal etiology is rarer in the temperate United States (just $1.2 \%$ of keratitis cases are in New York), but becomes more common in the sub-tropical regions (16-24\% in south Florida) [6,7]. Between 2005-2006, however, the Centers for Disease Control reported $>250$ cases of fungal keratitis in the U.S. and Asia associated with the Bausch and Lomb's ReNU with MoistureLoc contact lens solution [8]. This outbreak challenged epidemiologic norms in that it occurred in patient groups (contact lens wearers) and regions (the temperate U.S.) previously thought to be at low risk for fungal keratitis. Approximately $34 \%$ of patients in the outbreak required corneal transplantation, thus underscoring the severity of these infections even in areas in which antifungals are readily available [8].

Fusarium species have emerged as predominant agents of FK worldwide, accounting for more than half of all fungal corneal ulcers in south India and every case in the above-mentioned Renu outbreak $[4,8]$. This is a large and diverse genus, however, consisting of least twenty monophyletic clades (called "species complexes") that are distinguishable by molecular genotyping $[9,10]$. Sequencing of the internal transcribed spacer (ITS), situated between the $18 \mathrm{~S}$ and $5.8 \mathrm{~S}$ rRNA loci, can identify isolates at the species complex-level and has revealed that members of the Fusarium solani Species Complex (FSSC) account for 75-85\% of Fusarium keratitis cases overall [11]. In a recent analysis of Fusarium keratitis cases in Florida, FSSC infections were also found to require longer treatment course, have worse follow-up visual acuity (BCVA), and have higher rates of corneal transplantation than non-FSSC infections [12]. These data therefore suggest that FSSC members are both the most prevalent and most virulent agents of Fusarium keratitis [13].

Sequence variation at the translation elongation factor-1 (tef-1) locus can be used to place FSSC members into distinct molecular clades, some of which have been assigned separate species names. FSSC-clade 1, for example, is now called F. petroliphilum (sometimes F. solani var. petroliphilum), FSSC-2 is F. keratoplaticum, and FSSC-3 + 4 is F. falciforme [14-16]. The prevalence of these clades in the context of FK varies based on geography and etiology [17]. In south India, for example, F. falciforme accounts for $\sim 80 \%$ of all FSSC keratitis cases [18]. This organism is also the most common Fusarium species isolated from soil and vegetation, which fits with FK resulting from agricultural-related trauma [19]. By contrast, F. keratoplasticum and F. petroliphilum are among the primary agents of Fusarium keratitis in non-tropical climates and were predominant in the Moisture Loc outbreak [14,20]. These species are common isolates in plumbing systems, which may correlate with their association with contact lens infections via tap water contamination [10,21].

Despite the clear importance of FSSC pathogens in the context of FK, little is known about the genes involved in their corneal virulence. The most powerful research approach toward this end is that of reverse genetics, in which candidate genes are targeted (e.g., deleted) followed by virulence testing of the mutant in an FK model. The lack of FSSC research of this sort likely reflects two related issues. First is an intrinsically low targeting efficiency due to a non-homologous end joining (NHEJ) machinery that promotes ectopic integration of the DNA construct (i.e., high false-positive ratio) [22]. The second is a lack of robust transformation protocols that, when coupled with the first issue, necessitates the screening of perhaps hundreds of colonies obtained from multiple, laborious transformation attempts. These problems have been overcome in other fungi by a myriad of CRISPR-based methodologies, but none have so far been developed in FSSC organisms. This disparity is the focus of our report.

Clustered, regularly interspaced, short palindromic repeat (CRISPR) technologies require two critical components: 1) A CRISPR-associated (Cas9) endonuclease and, 2) a synthetic guide RNA (gRNA) - itself a fusion between CRISPR RNA (crRNA) and trans-activating crRNA (tracrRNA) - that associates with and directs Cas9 to a 20 nucleotide genomic target [23]. When this genomic target is directly next to protospacer-adjacent motif (PAM), typically an -NGG- sequence, Cas9 will induce a double-stranded break that can be repaired in one of several ways [24]. In the simplest iteration of the technique, the NHEJ machinery repairs the break within the gene's open reading frame, but in doing so introduces an indel mutation that results in a frameshift [25]. The Cas 9 cut can also be repaired by homologous recombination machinery when a "repair template" is introduced along with the CRISPR 
components [24]. In this way, the repair template may be a mutated or tagged allele that replaces the wild-type gene in situ. A third method, termed microhomology-mediated end joining (MMEJ), involves the introduction of short homology arms ( $40 \mathrm{bp})$ on either side of a selectable marker (repair template) for directed gene replacement [26,27].

In the following work, we employ an in vitro assembled Cas9 ribonucleoprotein (RNP) complex coupled with a MMEJ repair template for targeted gene replacement in an FSSC keratitis isolate [27]. We target a putative ura3 ortholog, encoding orotidine- $5^{\prime}$-phosphate decarboxylase, as a case study and demonstrate that $10 \%$ of transformants display the expected phenotype and gene replacement event. We further demonstrate that the use of CRISPR increases the transformation yield overall, providing more colonies that can be screened and increasing the likelihood of isolating the desired mutant. Taken together, this methodology represents a simple and efficient means for targeted gene replacement in a clinical FSSC isolate, which should bolster reverse genetic studies in these important but understudied pathogens.

\section{Materials and Methods}

\subsection{Fungal Isolates and Culture Media}

All experiments were conducted with a clinical FK isolate (06-0110) from UCSF, previously identified as FSSC based on ITS sequencing [28]. Species-level identification was carried out by amplifying the tef-1 locus with universal primers [29]. The PCR amplicon was subject to Sanger sequencing and the sequence was used in a BLASTn search against the Fusarium-ID database (isolate.fusariumdb.org). These results yielded a top hit for FSSC-1 (Fusarium petroliphilum).

Strains were cultured on glucose minimal medium (GMM) $(1 \%$ glucose, clutterbuck salts, hunters trace elements, $\mathrm{pH} 6.5$ ) or yeast, peptone, and dextrose medium (YPD; $2 \%$ dextrose, $2 \%$ peptone, $1 \%$ yeast extract) at $30^{\circ} \mathrm{C} .5 \mathrm{mM}$ of uracil and uridine were added when specified. $2 \mathrm{~g} / \mathrm{L}$ of 5-FOA was used in specified transformation selection media, and $1 \mathrm{~g} / \mathrm{L}$ was used for further phenotyping [30]. Hygromycin B was used at a concentration of $200 \mu \mathrm{g} / \mathrm{mL}$ when necessary. Conidia were harvested and washed using PBS.

\subsection{Design of crRNA's for ura3 in F. solani var petroliphilum}

The amino acid sequence for PyrG in Aspergillus nidulans was used as a query in a BLASTp against the Nectria haematococca v2.0 Necha2_best_proteins database. It should be noted that Nectria haematococca is the teleomorphic name for Fusarium solani. This search returned one high homology hit with an E value of $3.3 \times 10^{-53}$, Necha2199952, henceforth referred to as Ura3 the genomic sequence along with $500 \mathrm{bp}$ flanking sequences were downloaded and used for further analysis and the design of crRNAs and primers.

\subsection{Construction and Amplification of the Hygromycin Resistance Cassette}

A hygromycin B phosphotransferase expression cassette was used as the selectable marker for the Cas9-mediated gene deletion throughout this work. A 2100 bp product spanning the gpdA promoter, the hygromycin B phosphotransferase gene, and the $\operatorname{tr} p C$ terminator was amplified using Phusion polymerase (New England Biolabs, USA) from the plasmid pAN7 [31] using primers 1 and 2 (Table 1). The PCR fragments were purified using the E.Z.N.A Cycle Pure Kit and eluted in nuclease free water. The purified products were used as the microhomology mediated repair templates and contained a hygromycin B resistance cassette $(h y g R)$ flanked by $40 \mathrm{bp}$ sequences homologous to the up and downstream regions of the targeted ura3 gene. All primers used in this study are referenced in Table 1. 
Table 1. Oligonucleotides and crRNA's used for this study.

\begin{tabular}{|c|}
\hline Amplification of Hygromycin Resistance Cassette with Ura3 Microhomology \\
\hline $\begin{array}{c}\text { Primer 1: } \\
\text { 5' GCCTTGTCGCCTCTCGTAGCCGGCCGGTCCCTTGGAGCTGaagtggaaaggctggtgtgc } \\
\text { Primer 2: } \\
\text { 5’ AGTGGGGGCTCCGGAGTAATCGGCTTTAACGCAACCCACCtcgcgtggagccaagagcgg }\end{array}$ \\
\hline Genotyping putative $u r a 3$ deletants \\
\hline $\begin{array}{l}\text { Primer 3: 5' CAAGCCAAGCTTCGCACAAG } \\
\text { Primer 4: 5' GCGATGACATTCAGTGCAGC } \\
\text { Primer 5: 5' GTCGAGCTGCAATACACCAG } \\
\text { Primer 6: 5' GACAAGACGTGGTGAATCGG } \\
\text { Primer 7: 5' AAGTGGAAAGGCTGGTGTGC } \\
\text { Primer 8: 5' TCGCGTGGAGCCAAGAGCGG }\end{array}$ \\
\hline crRNA sequences \\
\hline $\begin{array}{l}\text { 5' crRNA FsUra3: 5' CGGCCGGTCCCTTGGAGCTG } \\
3^{\prime} \text { crRNA FsUra3: 5' GGGTTGAGTTTTGCGGTGGT }\end{array}$ \\
\hline
\end{tabular}

\subsection{Cas9-gRNA Ribonucleoprotein Complexes}

In Vitro assemblies were done as previously described in Al Abdallah et al. Briefly, Cas9 RNPs are composed of crRNA, tracrRNA, and the Alt-RßS.p. Cas9 Nuclease V3 (IDT 1081058) [27]. The gRNAs were assembled in vitro using $100 \mu \mathrm{M}$ stock solutions of the lyophilized crRNA (Table 1) and the tracrRNA were prepared in nuclease free duplex buffer (Integrated DNA Technologies, USA) and combined in equimolar amounts, resulting in final concentrations of $33.3 \mu \mathrm{M}$ concentrations of each [27]. This solution was heated to $95^{\circ} \mathrm{C}$ for $5 \mathrm{~min}$ and allowed to cool to room temperature $\left(20^{\circ} \mathrm{C}\right)$ for $20 \mathrm{~min}$. The resulting gRNAs were stored on ice for the short term and at $-20^{\circ} \mathrm{C}$ long-term.

In order to form the Cas9 RNP $1.5 \mu \mathrm{L}$ of each gRNA were separately combined with $0.75 \mu \mathrm{g}$ of Cas9 in $11 \mu \mathrm{L}$ of nuclease free Cas9 working buffer (20 mM HEPES, $150 \mathrm{mM} \mathrm{KCl}, \mathrm{pH} 7.5)$. This mixture was incubated at $37^{\circ} \mathrm{C}$ for $5 \mathrm{~min}$ to allow each separate Cas9 RNP to form before they were mixed (bringing the final volume to $\sim 26.5 \mu \mathrm{L}$ ) and added to the F. petroliphilum protoplasts.

\subsection{PEG-Mediated Fungal Transformation}

Transformations were performed as previously described by Yelton et al. and Al Abdallah et al. with some modification [27,32]. Approximately $1 \times 10^{8}$ microconidia were inoculated into YPD broth and germinated at $28^{\circ} \mathrm{C}$ at $160 \mathrm{rpm}$ overnight. The germlings were collected in miracloth and resuspended in $1.2 \mathrm{M} \mathrm{KCl}$. The germlings were treated with a mixture of $5 \mathrm{mg} / \mathrm{mL}$ of Lysing Enzymes from Trichoderma harzianum (SIGMA), $5 \mathrm{mg} / \mathrm{mL}$ of Driselase (SIGMA, USA) and $100 \mu \mathrm{g} / \mathrm{mL}$ of Chitinase from Streptomyces griseus (SIGMA, USA). The germling and enzyme mixture was incubated at $28^{\circ} \mathrm{C}$ for $2 \mathrm{~h}$ at $50 \mathrm{rpm}$. This suspension was monitored every $30 \mathrm{~min}$ microscopically for the formation of protoplasts.

Protoplasts were collected after $2 \mathrm{~h}$ incubation by centrifugation ( $5 \mathrm{~min}$ at $4000 \mathrm{~g}$ at $4{ }^{\circ} \mathrm{C}$ ) and washed twice in STC50 buffer (1.2 M Sorbitol, $10 \mathrm{mM} \mathrm{CaCl}_{2}, 10 \mathrm{mM}$ Tris $\mathrm{HCl} \mathrm{pH}$ 7.5). The protoplasts were resuspended in $1 \mathrm{~mL}$ of STC50 and enumerated on a hemocytometer, and diluted to a concentration of $2 \times 10^{7} .200 \mu \mathrm{L}$ of the protoplasts were used for each transformation.

$26.5 \mu \mathrm{L}$ of the Cas9 RNPs and $10 \mu \mathrm{g}$ of the repair template were combined with $200 \mu \mathrm{L}$ of protoplasts and $25 \mu \mathrm{L}$ of a $60 \%$ PEG solution $(60 \%(w / v)$ PEG MW 3350, $50 \mathrm{mM} \mathrm{CaCl} 2,50 \mathrm{mM}$ Tris $\mathrm{HCl} \mathrm{pH} \mathrm{7.5)} \mathrm{and} \mathrm{gently} \mathrm{mixed} \mathrm{by} \mathrm{pipetting.} \mathrm{This} \mathrm{solution} \mathrm{was} \mathrm{placed} \mathrm{on} \mathrm{ice} \mathrm{for} 50 \mathrm{~min} .1 .25 \mathrm{~mL}$ of the $60 \%$ PEG solution was added and mixed by gently swirling and incubated at room temperature for $20 \mathrm{~min}$. $1 \mathrm{~mL}$ of STC50 was added to each transformation and $150 \mu \mathrm{L}$ were plated onto GMM, 1.2 M Sorbitol, $5 \mathrm{mM}$ of Uracil and Uridine, 1.5\% Agar, and $2 \mathrm{~g} / \mathrm{L}$ of 5-FOA when indicated. After an overnight incubation $200 \mu \mathrm{g} / \mathrm{mL}$ of hygromycin B was introduced in an overlay (GMM with $0.5 \%$ 
Agar and 5mM Uracil and Uridine). Colonies were picked from the primary transformation plate onto minimal media supplemented with hygromycin, uracil, and uridine over the next 5-10 days of incubation at $30^{\circ} \mathrm{C}$. All genotyping PCRs, ed using NEB Hot Start Taq 2x Mastermix (New England Biolabs, USA) using the primers listed in Table 1 and the Figure 3 legend.

\section{Results}

\subsection{Design of the crRNA Protospacer Sequences for Replacement of the ura3 Gene with a Hygromycin} Resistance Cassette

Our overall goal was to test the feasibility of using a CRISPR/Cas9 system for targeted gene replacement in a corneal isolate from the U.S., identified initially as Fusarium by culture and then placed within the FSSC by ITS sequencing [28]. For this study, we further identified the isolate as FSSC 1 (F. petroliphilum) by performing a BLASTn of the tef-1 locus against the Fusarium-ID database. Accordingly, we will now refer to this organism as F. petroliphilum.

As a case study, we chose to target the ura3 gene that, in other fungi, encodes the orotodine-5'-phosphate decarboxylase enzyme essential for uracil biosynthesis (Figure 1) [33]. The enzyme also converts exogenously added 5-fluoroorotic acid (5-FOA) to a toxic metabolite $5^{\prime}$-fluorouracil [34]. Accordingly, the successful replacement of the ura3 gene with the $h y g R$ cassette would result in three easily assayable phenotypes: 1) Hygromycin resistance, 2) uracil auxotrophy, and 3) 5'-FOA resistance. To begin, we first identified the ura3 ortholog in F. petroliphilum through a BLASTp search of the Nectria haemotococca v2.0 database (Mycocosm, Joint Genome Institute) using the A. nidulans ortholog, PyrG, as a query $[35,36]$. This yielded a single hit with $54.7 \%$ sequence identity (Necha2199952), henceforth referred to as ura3.

We sought to employ an in vitro CRISPR-MMEJ method in which two components are introduced into fungal protoplasts $[26,27,37]$. First is the Cas9 ribonucleoprotein (RNP) complex, which consists of a commercially available Cas9 protein coupled with a gRNA. With this approach, the gRNA is assembled in vitro from two components: An invariable tracrRNA (also purchased commercially) and a target-specific CRISPR RNA (crRNA). Two distinct crRNAs were designed against genomic sequences (protospacers) within $100 \mathrm{bp}$ of the putative ura3 start and stop codons (UTRs). These protospacers (20 bp) not only harbored a PAM site (-NGG) at one end, as is essential, but they also contained one or more PAM sites within them as this has been suggested to increase Cas9 cutting efficiency and specificity (Figure 1C) [38]. Potential protospacer sequences were used as a query for a BLASTn search against the genome database to determine the possibility of any off-target cutting. In this way, protospacers that displayed more than $15 \mathrm{bp}$ homology with a sequence preceding an off-target PAM motif were not used [39]. The second component required for the Cas9-MMEJ protocol is the repair template. Microhomology (40 bp) flanks, designed adjacent to the PAM site and crRNA protospacers, were incorporated into the hygR cassette by PCR. The design of both the microhomology flanks and the crRNA protospacers are illustrated in Figure 1C and the primer sequences are listed in Table 1.

In short, two Cas9 RNPs were generated in vitro such that cutting would occur in ura3 $5^{\prime}$ and $3^{\prime}$ UTRs. These cuts would, in principle, promote the replacement of the ura 3 coding sequence with the repair template by homologous recombination via the microhomology flanks. 
A

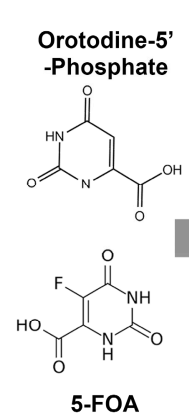

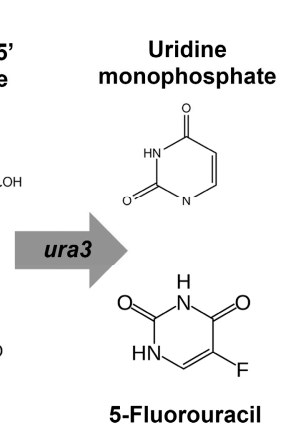

B

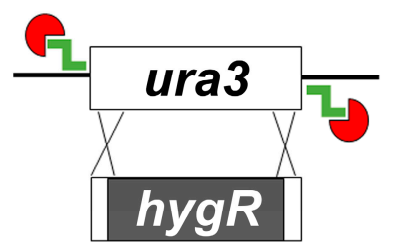

$\Delta$ ura3 phenotypes:

- hygromycin resistant

- 5' FOA resistant

- uracil auxotroph

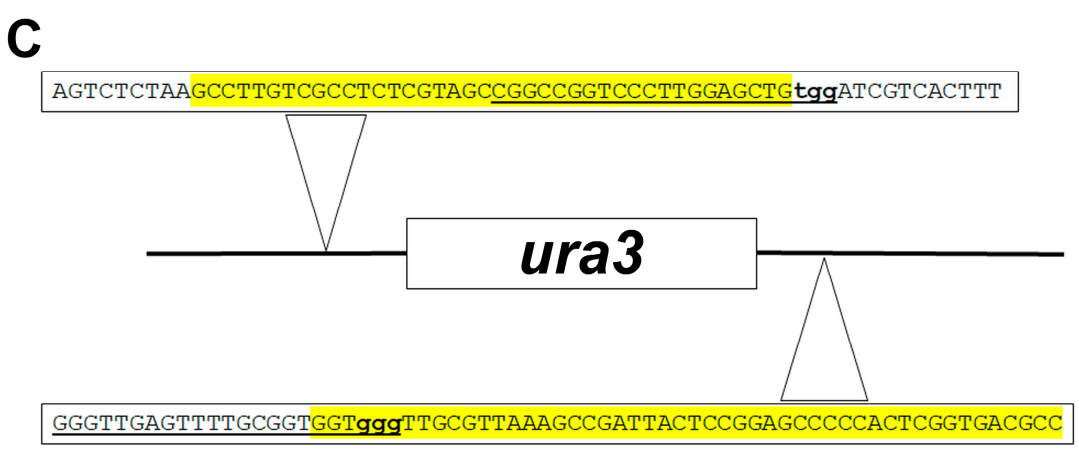

Figure 1. An overview of the ura3 deletion strategy using in vitro assembled Cas9 RNPs coupled with microhomology-mediated end joining (MMEJ). (A) Ura3 decarboxylates orotodine-5' $5^{\prime}$-monophosphate to form uridine monophosphate, an essential uracil precursor, as well as 5-fluorotic acid (FOA), added exogenously, to form a toxic metabolite 5-fluorouracil. (B) Cas9 ribonucleoprotein (RNP)'s target the up and downstream regions of the $u r a 3$ gene and the MMEJ repair replaces ura3 with hygR resulting in uracil auxotrophy, 5-FOA resistance, and hygromycin resistance. (C) The regions of microhomology for the repair template are highlighted in yellow, the crRNA sequences are underlined, and the protospacer-adjacent motif (PAM) sites are bolded in lowercase.

\subsection{Cas9-MMEJ Transformation Results in Several Colonies of the Expected ura3 Deletion Phenotype}

To analyze the efficacy of the above-described CRISPR strategy, three F. petroliphilum protoplast transformation groups were analyzed: (1) Control, which received no Cas9 RNPs or repair template DNA; (2) repair template only, which received $10 \mu$ g of repair template DNA; and (3) Cas9-MMEJ, which received both $10 \mu \mathrm{g}$ of the repair template and the two flanking Cas9 RNP complexes. Protoplasts from each group were initially recovered on, and then subcultured onto, minimal media supplemented with hygromycin, uracil and uridine. In this way, stable transformants would grow regardless of whether the repair template integrated homologously or ectopically. As reflected in Table 2, the total number of hygromycin resistant colonies was $\sim 5$ times higher in the Cas9-MMEJ group compared to the repair template only group, suggesting that the Cas9 RNPs increased the transformation efficiency in some way. As expected, the control group yielded no hygromycin-resistant colonies.

Table 2. Results of the PEG mediated transformation. A subset of hygromycin resistant colonies were subcultured onto various media. Uracil auxotrophs could not grow on media that did not contain uracil.

\begin{tabular}{cccccc}
\hline & $\begin{array}{c}\mathbf{1}^{\circ} \text { Plate (Hyg } \\
\text { Selection) }\end{array}$ & Analyzed & Hyg Resistant & $\begin{array}{c}\text { Uracil } \\
\text { Auxotrophs }\end{array}$ & Efficiency \\
\hline Control & 0 & 0 & 0 & 0 & $0 \%$ \\
Repair Template only & 16 & 10 & 10 & 0 & $0 \%$ \\
Cas9-MMEJ & 83 & 63 & 63 & 6 & $9.50 \%$ \\
\hline
\end{tabular}


To determine if any of the hygromycin-resistant transformants were also uracil auxotrophs, the colonies were subcultured onto minimal media supplemented with either (1) uracil/uridine, (2) hygromycin and uracil/uridine, (3) neither. In addition to the transformants, the wild-type strain was included on each medium as a control. Figure 2 shows a subset of the Cas9-MMEJ transformants screened in this way, all of which were able to grow in the presence of hygromycin as expected. Six colonies of those screened $(\sim 10 \%)$ were unable to grow in the absence of uracil/uridine, indicating that they were ura3 deletants (auxotrophs). By contrast, none of the "repair template only" transformants were uracil auxotrophs, suggesting that they all had the $h y g R$ cassette present as an ectopic integration (Table 2, and data not shown).

A

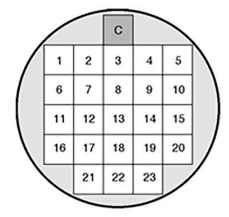

Hygromycin B: Uracil: Uridine:

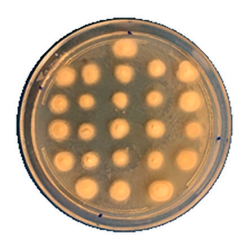

$-$

$+$

$+$

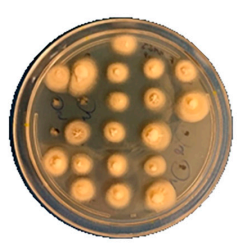

-

$-$

B

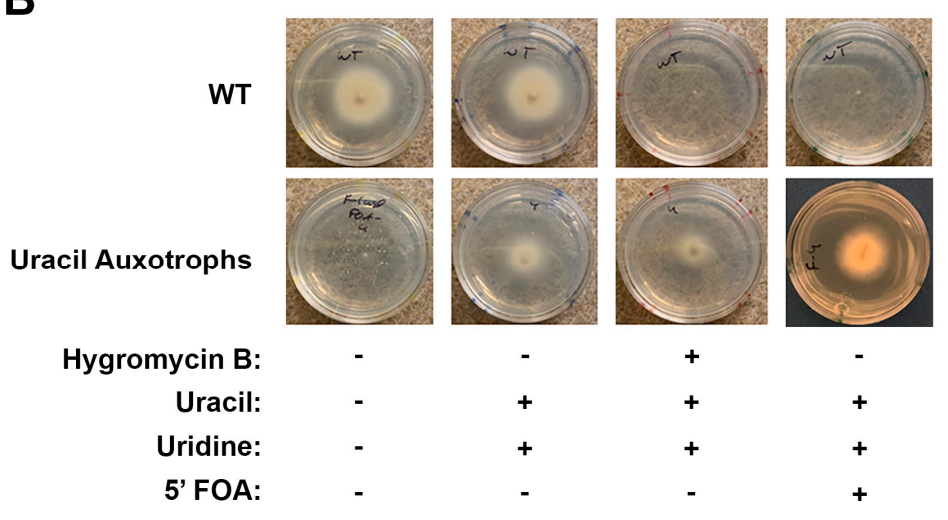

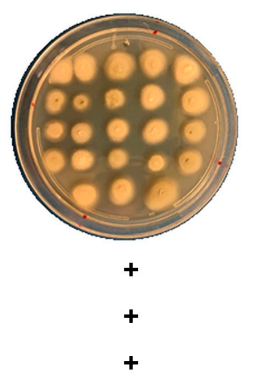

Figure 2. Phenotyping transformants for uracil auxotrophy and 5-FOA resistance. (A) Transformants were picked and replicated across three growth conditions with each position on the plate corresponding to a single transformant. The topmost position is the wild-type control. Transformants in positions 6, 7, 11, 15 and 20 displayed the desired uracil auxotrophy phenotype (center plate). (B) A subculture of an uracil auxotroph as compared to the wild type control. Incubation times varied from 5-10 days at $30{ }^{\circ} \mathrm{C}$. Incubation on Media containing 5-FOA needed 10 days for comparable growth to media without 5-FOA.

In addition to being auxotrophic for uracil/uridine, ura3 deletion mutants should also be resistant to 5-FOA. Indeed, whereas the recipient wild-type strain was completely growth inhibited on 5-FOA-containing medium, the uracil/uridine auxotrophs grew otherwise normally after a brief delay (Figure 2B). Taken together, the phenotypic data suggests that a proportion of the hygromycin-resistant transformants recovered from the Cas9-MMEJ transformation were the desired ura3 replacements.

\subsection{Genotyping of the Uracil Auxotrophic Mutants Confirms the Expected ura3 Gene Replacement Event}

To characterize the genotype of the uracil auxotrophs, we first performed a PCR of the ura3 locus with primers (primers $\frac{3}{4}$, Table 1) situated outside of the expected area of recombination(Figure 3A). The repair template is approximately $100 \mathrm{bp}$ larger than the ura3 coding sequence and, as expected, this band shift was apparent in all of the auxotrophic mutants analyzed. We then used the ura3 locus 
amplicon (Figure 3A) as a template for another PCR with nested primers (primers 7/8, Table 1), for the $h y g R$ cassette. All auxotrophic mutants yielded a band in this assay, indicating that the ura 3 coding sequence was replaced with the $h y g R$ cassette (Figure $3 \mathrm{~B}$ ). To confirm that the ura3 coding sequence was indeed absent from the genome in these mutants, we next performed a PCR on the genomic DNA with primers situated within the coding region (primers 5/6, Table 1). Again, as expected, the wild-type strain yielded a predicted amplicon whereas the auxotrophic mutants did not (Figure 3C).

Cumulatively, these results indicate that the auxotrophic phenotypes observed in our Cas9-MMEJ experiment were due to the expected recombination event.

A
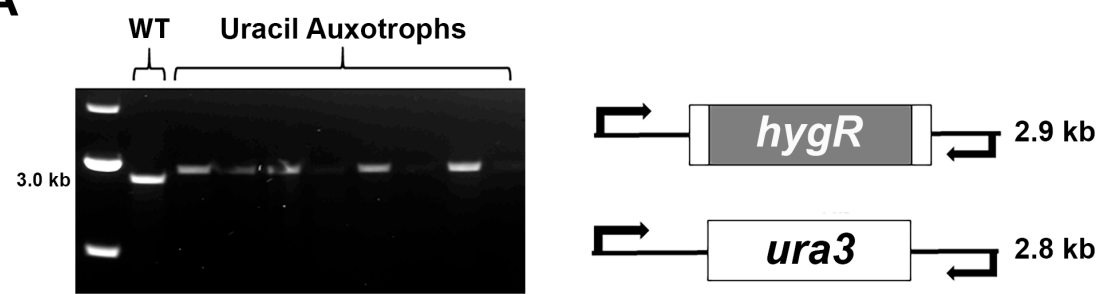

B
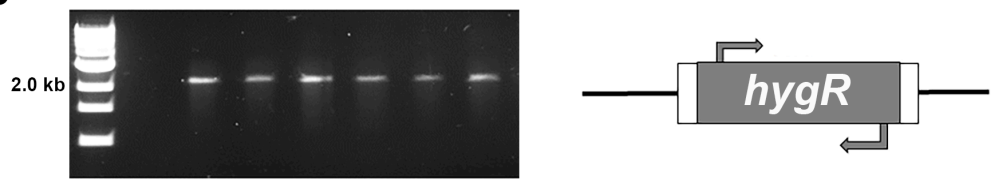

$2.0 \mathrm{~kb}$
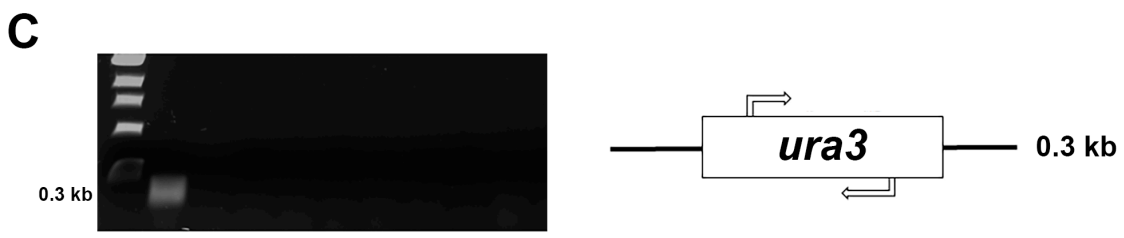

Figure 3. Genotyping of uracil auxotrophs. (A) Amplification of the ura3 locus using primers 3 and 4 yields the expected $100 \mathrm{bp}$ band shift between the wild-type and the uracil auxotrophs. (B) Amplification of the $h y g R$ cassette from the amplicon in Figure $3 \mathrm{~A}$ using primers 7 and 8. The uracil auxotrophs contain the hygromycin resistance cassette while the wild-type strain does not. (C) Amplification of the ura3 coding region from genomic DNA using primers 5 and 6 . Only the wild-type contains the coding sequence for ura3.

\section{Discussion}

The early CRISPR systems developed in fungi involved the integration of cas 9 and single guide (sg)RNA cassettes in the organism's genome [40]. In the absence of a repair template, gene disruption is dependent upon the error-prone NHEJ machinery to induce nonsense mutations or otherwise non-functional gene products [41]. By contrast, the inclusion of MHEJ repair templates allowed for a greater versatility of applications, including site-directed mutagenesis and in situ gene tagging [42]. The presence of such repair templates also increases the overall targeting efficiency of the system. The Cas9-mediated disruption of $p k s P$ in $A$. fumigatus, for example, increased from approximately $45 \%$ by NHEJ to $95-100 \%$ with the inclusion of a MMEJ repair template [43]. In any case, such methodologies raise an important concern of whether the presence of the cas 9 gene within the cell would have long-term, genotoxic effects. Indeed, it is estimated that gRNAs, on average, can have up to five mismatch (off-target) genes, and the constitutive presence of cas 9 likely increases the probability they will be mutated $[44,45]$. Although Fuller et al. demonstrated that constitutive expression of cas 9 did not impact growth or virulence of $A$. fumigatus, Foster et al. recently reported that cas 9 expression was highly toxic to Magnaporthe oryzae $[37,46]$. This suggests that Cas 9 can mediate unintended and deleterious mutations, at least in some fungal species. In attempt to mitigate such effects, conditional 
promoter systems have been developed, such as $p$ NiiA in A. fumigatus, as well as "suicide system" in Cryptococcus neoformans in which the cas 9 cassette is excised from the genome after editing [43,47].

The in vitro-assembled Cas9 approach, in principle, largely bypasses the concern of long-term cas 9 toxicity and off-target effects $[46,48]$. As this system involves the transient introduction of purified Cas9 RNPs into protoplasts, there is no genetic material that can be integrated into the genome beyond the repair template. Indeed, Abdallah et al. performed whole-genome sequencing of A. fumigatus mutants generated by this approach and found that Cas9 RNPs did not induce more secondary mutations than traditional homologous recombination techniques [48]. The activity of Cas9 may vary between species, however, and so our future studies will employ whole-genome sequencing in this and other CRISPR-mediated F. petroliphilum mutants to determine the cryptic mutation rate.

A further advantage of the in vitro systems is their ease of use. Each of the in vitro-assembled RNP components may be obtained commercially, so the only molecular biology required ahead of transformation is PCR amplification of the selectable marker (repair template) with primers that incorporate the MMEJ flanks. The resulting PCR amplicon can then be used directly in the transformation, thus negating the need for any cloning step.

In this study, an in vitro-assembled Cas9-MMEJ system was used to successfully replace the ura3 ortholog in a F. petroliphilum corneal isolate. Based on screening hygromycin-resistant transformants for uracil auxotrophy followed by genotyping, we describe a gene targeting efficiency of $10 \%$. This efficiency is relatively low compared to some fungal wild-type backgrounds in which a similar CRISPR strategy was employed, including A. fumigatus (50-75\%), Magnaporthe oryzae (70-80\%), and C. neoformans $(81-87 \%)[27,46,47]$. However, our rates are comparable with other fungal species, including Fusarium oxysporum (21\%) and Trichoderma reesei (3-30\%), suggesting that there is a strong species-dependent influence on targeting efficiency $[26,49]$. The presence of a robust NHEJ machinery likely accounts for ectopic integration of the repair template and this can be overcome by utilizing backgrounds deficient in essential NHEJ genes. For example, the targeting of $p k s P$ in $A$. fumigatus increased from $50-75 \%$ in a wild-type strain to $95-100 \%$ in an $\triangle a k u B$ (ku80) mutant [27]. However, the power of CRISPR, at least for our interests, lies within the ability to transform clinical isolates. We noticed that the inclusion of Cas9 RNPs increased the number of hygromycin-resistance colonies overall five-fold, from 16 in the "template only" group to 83 . Therefore, a $10 \%$ targeting efficiency makes it probable that several mutants of interest will be isolated within a single transformation attempt. Future efforts will focus on optimizing the protocol by adjusting the MMEJ homology length and/or Cas9 RNP concentrations.

In summary, we report the first use, to our knowledge, of CRISPR/Cas9 technology for reverse genetics in F. petroliphilum or any FSSC species. An appreciation of the FSSC in the context of keratitis and systemic fusariosis has increased in recent years, but an understanding of genes that govern their pathogenesis has not. With improved genetic systems in place, including the in vitro Cas9-MMEJ system described here, we hope to bolster virulence-related research in these important pathogens and improve treatment modalities in the patients affected by them.

Author Contributions: Conceptualization, methodology and investigation, J.D.L. and K.K.F.; resources and funding acquisition, K.K.F.; writing—original draft preparation, J.D.L. and K.K.F.; writing—review and editing, J.D.L. and K.K.F.

Funding: This work was supported by a grant from the National Eye Institute, NIH (R21 EY028677) and a Career Development Award from Research to Prevent Blindness, both to K.K.F.

Acknowledgments: We thank the Fungal Genetics Stock Center (Manhattan, Kansas) for its critical and ongoing support to fungal research community. We thank Robert Cramer at Dartmouth for the gift of the Fusarium clinical isolate used in this study.

Conflicts of Interest: The authors declare no conflict of interest.

\section{References}

1. Leal, S.M.; Pearlman, E. The role of cytokines and pathogen recognition molecules in fungal keratitis-Insights from human disease and animal models. Cytokine 2012, 58, 107-111. [CrossRef] [PubMed] 
2. Prajna, N.V.; Srinivasan, M.; Lalitha, P.; Krishnan, T.; Rajaraman, R.; Ravindran, M.; Mascarenhas, J.; Oldenburg, C.E.; Ray, K.J.; McLeod, S.D.; et al. Differences in clinical outcomes in keratitis due to fungus and bacteria. JAMA Ophthalmol. 2013, 131, 1088-1089. [CrossRef] [PubMed]

3. Ansari, Z.; Miller, D.; Galor, A. Current thoughts in fungal keratitis: Diagnosis and treatment. Curr. Fungal Infect. Rep. 2013, 7, 209-218. [CrossRef] [PubMed]

4. Homa, M.; Shobana, C.S.; Singh, Y.R.B.; Manikandan, P.; Selvam, K.P.; Kredics, L.; Narendran, V.; Vágvölgyi, C.; Galgóczy, L. Fusarium keratitis in South India: Causative agents, their antifungal susceptibilities and a rapid identification method for the Fusarium solani species complex. Mycoses 2013, 56, 501-511. [CrossRef] [PubMed]

5. Godoy, P.; Colombo, A.L.; Höfling-Lima, A.L.; Cano, J.; Gené, J.; Guarro, J. Genotyping of 44 isolates of Fusarium solani, the main agent of fungal keratitis in Brazil. J. Clin. Microbiol. 2004, 42, 4494-4497. [CrossRef]

6. Rosa, R.H.; Miller, D.; Alfonso, E.C. The changing spectrum of fungal keratitis in South Florida. Ophthalmology 1994, 101, 1005-1013. [CrossRef]

7. Ritterband, D.C.; Seedor, J.A.; Shah, M.K.; Koplin, R.S.; McCormick, S.A. Fungal keratitis at the New York eye and ear infirmary. Cornea 2006, 25, 264-267. [CrossRef]

8. Chang, D.C.; Grant, G.B.; O’Donnell, K.; Wannemuehler, K.A.; Noble-Wang, J.; Rao, C.Y.; Jacobson, L.M.; Crowell, C.S.; Sneed, R.S.; Lewis, F.M.T.; et al. Multistate outbreak of Fusarium keratitis associated with use of a contact lens solution. J. Am. Med. Assoc. 2006, 296, 953-963. [CrossRef]

9. Ma, L.-J.; Geiser, D.M.; Proctor, R.H.; Rooney, A.P.; O’Donnell, K.; Trail, F.; Gardiner, D.M.; Manners, J.M.; Kazan, K. Fusarium Pathogenomics. Annu. Rev. Microbiol. 2013, 67, 399-416. [CrossRef]

10. Short, D.P.; O'Donnell, K.; Geiser, D.M. Clonality, recombination, and hybridization in the plumbing-inhabiting human pathogen Fusarium keratoplasticum inferred from multilocus sequence typing. BMC Evol. Biol. 2014, 14, 91. [CrossRef]

11. Debourgogne, A.; Gueidan, C.; de Hoog, S.; Lozniewski, A.; Machouart, M. Comparison of two DNA sequence-based typing schemes for the Fusarium solani species complex and proposal of a new consensus method. J. Microbiol. Methods 2012, 91, 65-72. [CrossRef] [PubMed]

12. Oechsler, R.A.; Feilmeier, M.R.; Miller, D.; Shi, W.; Hofling-Lima, A.L.; Alfonso, E.C. Fusarium keratitis: Genotyping, in vitro susceptibility and clinical outcomes. Cornea 2013, 32, 667-673. [CrossRef] [PubMed]

13. Dallé da Rosa, P.; Nunes, A.; Borges, R.; Batista, B.; Meneghello Fuentefria, A.; Goldani, L.Z. In Vitro susceptibility and multilocus sequence typing of Fusarium isolates causing keratitis. J. Mycol. Med. 2018, 28, 482-485.

14. Short, D.P.G.; O'Donnell, K.; Thrane, U.; Nielsen, K.F.; Zhang, N.; Juba, J.H.; Geiser, D.M. Phylogenetic relationships among members of the Fusarium solani species complex in human infections and the descriptions of F. keratoplasticum sp. nov. and F. petroliphilum stat. nov. Fungal Genet. Biol. 2013, 53, 59-70. [CrossRef] [PubMed]

15. Coleman, J.J. The Fusarium solani species complex: Ubiquitous pathogens of agricultural importance. Mol. Plant Pathol. 2016, 17, 146-158. [CrossRef] [PubMed]

16. Herkert, P.F.; Al-Hatmi, A.M.S.; de Oliveira Salvador, G.L.; Muro, M.D.; Pinheiro, R.L.; Nucci, M.; Queiroz-Telles, F.; de Hoog, G.S.; Meis, J.F. Molecular characterization and antifungal susceptibility of clinical Fusarium species from Brazil. Front. Microbiol. 2019, 10, 737. [CrossRef] [PubMed]

17. Geiser, D.M.; Aoki, T.; Bacon, C.W.; Baker, S.E.; Bhattacharyya, M.K.; Brandt, M.E.; Brown, D.W.; Burgess, L.W.; Chulze, S.; Coleman, J.J.; et al. One fungus, one name: Defining the genus Fusarium in a scientifically robust way that preserves longstanding use. Phytopathology 2013, 103, 400-408. [CrossRef]

18. Homa, M.; Galgóczy, L.; Manikandan, P.; Narendran, V.; Sinka, R.; Csernetics, Á.; Vágvölgyi, C.; Kredics, L.; Papp, T. South Indian Isolates of the Fusarium solani species complex from clinical and environmental samples: Identification, antifungal susceptibilities, and virulence. Front. Microbiol. 2018, 9, 1052. [CrossRef]

19. Hassan, A.S.; Al-Hatmi, A.M.S.; Shobana, C.S.; Van Diepeningen, A.D.; Kredics, L.; Vágvölgyi, C.; Homa, M.; Meis, J.F.; De Hoog, G.S.; Narendran, V.; et al. Antifungal Susceptibility and Phylogeny of Opportunistic Members of the Genus Fusarium Causing Human Keratomycosis in South India. Med. Mycol. 2016, 54, 287-294. [CrossRef]

20. Walther, G.; Stasch, S.; Kaerger, K.; Hamprecht, A.; Roth, M.; Cornely, O.A.; Geerling, G.; Mackenzie, C.R.; Kurzai, O.; Von Lilienfeld-Toal, M. Fusarium keratitis in Germany. J. Clin. Microbiol. 2017, 55, 2983-2995. [CrossRef] 
21. Scheel, C.M.; Hurst, S.F.; Barreiros, G.; Akiti, T.; Nucci, M.; Balajee, S.A. Molecular analyses of Fusarium isolates recovered from a cluster of invasive mold infections in a Brazilian hospital. BMC Infect. Dis. 2013, 13, 49. [CrossRef] [PubMed]

22. Fernández-Martín, R.; Cerdá-Olmedo, E.; Avalos, J. Homologous recombination and allele replacement in transformants of Fusarium fujikuroi. Mol. Gen. Genet. 2000, 263, 838-845. [CrossRef] [PubMed]

23. Wang, S.; Chen, H.; Tang, X.; Zhang, H.; Chen, W.; Chen, Y.Q. Molecular tools for gene manipulation in filamentous fungi. Appl. Microbiol. Biotechnol. 2017, 101, 8063-8075. [CrossRef] [PubMed]

24. Sander, J.D.; Joung, J.K. CRISPR-Cas systems for editing, regulating and targeting genomes. Nat. Biotechnol. 2014, 32, 347-350. [CrossRef]

25. Nødvig, C.S.; Nielsen, J.B.; Kogle, M.E.; Mortensen, U.H. A CRISPR-Cas9 system for genetic engineering of filamentous fungi. PLoS ONE 2015, 10, e0133085. [CrossRef]

26. Wang, Q.; Cobine, P.A.; Coleman, J.J. Efficient genome editing in Fusarium oxysporum based on CRISPR/Cas9 ribonucleoprotein complexes. Fungal Genet. Biol. 2018, 117, 21-29. [CrossRef]

27. Al Abdallah, Q.; Ge, W.; Fortwendel, J.R. A simple and universal system for gene manipulation in Aspergillus fumigatus: In vitro-assembled Cas9-guide RNA ribonucleoproteins coupled with microhomology repair templates. mSphere 2017, 2, 1-14. [CrossRef]

28. Rees, C.A.; Bao, R.; Zegans, M.E.; Cramer, R.A. Natamycin and Voriconazole Exhibit Synergistic Interactions with nonantifungal ophthalmic agents against Fusarium species ocular isolates. Antimicrob. Agents Chemother. 2019, 63, e02505-e02518. [CrossRef]

29. Chehri, K.; Salleh, B.; Zakaria, L. Morphological and phylogenetic analysis of Fusarium solani species complex in malaysia. Microb. Ecol. 2015, 69, 457-471. [CrossRef]

30. Oakley, B.R. Aspergillus nidulans. In Brenner's Encyclopedia of Genetics: Second Edition; Springer: Boston, MA, USA, 2013; pp. 212-215. ISBN 9780080961569.

31. Ventura, L.; Ramón, D. Transformation of Aspergillus terreus with the hygromycin B resistance marker from Escherichia coli. FEMS Microbiol. Lett. 1991, 82, 189-193. [CrossRef]

32. Yelton, M.M.; Hamer, J.E.; Timberlake, W.E. Transformation of aspergillus nidulans by using a trpC plasmid. Proc. Natl. Acad. Sci. USA 1984, 81, 1470-1474. [CrossRef] [PubMed]

33. Van Hartingsveldt, W.; Mattern, I.E.; van Zeijl, C.M.J.; Pouwels, P.H.; van den Hondel, C.A.M.J.J. Development of a homologous transformation system for Aspergillus niger based on the pyrG gene. MGG Mol. Gen. Genet. 1987, 206, 71-75. [CrossRef] [PubMed]

34. Alani, E.; Cao, L.; Kleckner, N. A method for gene disruption that allows repeated use of URA3 selection in the construction of multiply disrupted yeast strains. Genetics 1987, 116, 541-545. [CrossRef] [PubMed]

35. Grigoriev, I.V.; Nikitin, R.; Haridas, S.; Kuo, A.; Ohm, R.; Otillar, R.; Riley, R.; Salamov, A.; Zhao, X.; Korzeniewski, F.; et al. MycoCosm portal: Gearing up for 1000 fungal genomes. Nucleic Acids Res. 2014, 42, D699-D704. [CrossRef]

36. Tani, S.; Tsuji, A.; Kunitake, E.; Sumitani, J.-I.; Kawaguchi, T. Reversible impairment of the ku80 gene by a recyclable marker in Aspergillus aculeatus. AMB Express 2013, 3, 4. [CrossRef]

37. Fuller, K.K.; Chen, S.; Loros, J.J.; Dunlap, C. Development of the CRISPR/Cas9 system for targeted gene disruption in Aspergillus fumigatus. Eukaryot Cell 2015, 14, 1073-1080. [CrossRef]

38. Sternberg, S.H.; Redding, S.; Jinek, M.; Greene, E.C.; Doudna, J.A. DNA interrogation by the CRISPR RNA-guided endonuclease Cas9. Nature 2014, 507, 62-67. [CrossRef]

39. Zheng, T.; Hou, Y.; Zhang, P.; Zhang, Z.; Xu, Y.; Zhang, L.; Niu, L.; Yang, Y.; Liang, D.; Yi, F.; et al. Profiling single-guide RNA specificity reveals a mismatch sensitive core sequence. Sci. Rep. 2017, 7, 40638. [CrossRef]

40. Song, R.; Zhai, Q.; Sun, L.; Huang, E.; Zhang, Y.; Zhu, Y.; Guo, Q.; Tian, Y.; Zhao, B.; Lu, H. CRISPR/Cas9 genome editing technology in filamentous fungi: Progress and perspective. Appl. Microbiol. Biotechnol. 2019, 103, 6919-6932. [CrossRef]

41. Shi, T.Q.; Liu, G.N.; Ji, R.Y.; Shi, K.; Song, P.; Ren, L.J.; Huang, H.; Ji, X.J. CRISPR/Cas9-based genome editing of the filamentous fungi: The state of the art. Appl. Microbiol. Biotechnol. 2017, 101, 7435-7443. [CrossRef]

42. Jan Vonk, P.; Escobar, N.; Wösten, H.A.B.; Lugones, L.G.; Ohm, R.A. High-throughput targeted gene deletion in the model mushroom Schizophyllum commune using pre-assembled Cas9 ribonucleoproteins. Sci. Rep. 2019, 9, 7632. [CrossRef] [PubMed] 
43. Zhang, C.; Meng, X.; Wei, X.; Lu, L. Highly efficient CRISPR mutagenesis by microhomology-mediated end joining in Aspergillus fumigatus. Fungal Genet. Biol. 2016, 86, 47-57. [CrossRef] [PubMed]

44. Hsu, P.D.; Scott, D.A.; Weinstein, J.A.; Ran, F.A.; Konermann, S.; Agarwala, V.; Li, Y.; Fine, E.J.; Wu, X.; Shalem, O.; et al. DNA targeting specificity of RNA-guided Cas9 nucleases. Nat. Biotechnol. 2013, 31, 827-832. [CrossRef] [PubMed]

45. Pattanayak, V.; Lin, S.; Guilinger, J.P.; Ma, E.; Doudna, J.A.; Liu, D.R. High-throughput profiling of off-target DNA cleavage reveals RNA-programmed Cas9 nuclease specificity. Nat. Biotechnol. 2013, 31, 839-843. [CrossRef]

46. Foster, A.J.; Martin-Urdiroz, M.; Yan, X.; Wright, H.S.; Soanes, D.M.; Talbot, N.J. CRISPR-Cas9 ribonucleoprotein-mediated co-editing and counterselection in the rice blast fungus. Sci. Rep. 2018, 8, 14355. [CrossRef]

47. Wang, Y.; Wei, D.; Zhu, X.; Pan, J.; Zhang, P.; Huo, L.; Zhu, X. A “suicide” CRISPR-Cas9 system to promote gene deletion and restoration by electroporation in Cryptococcus neoformans. Sci. Rep. 2016, 6, 31145. [CrossRef]

48. Al Abdallah, Q.; Souza, A.C.O.; Martin-Vicente, A.; Ge, W.; Fortwendel, J.R. Whole-genome sequencing reveals highly specific gene targeting by in vitro assembled Cas9-ribonucleoprotein complexes in Aspergillus fumigatus. Fungal Biol. Biotechnol. 2018, 5, 11. [CrossRef]

49. Hao, Z.; Su, X. Fast gene disruption in Trichoderma reesei using in vitro assembled Cas9/gRNA complex. BMC Biotechnol. 2019, 19, 2. [CrossRef]

(C) 2019 by the authors. Licensee MDPI, Basel, Switzerland. This article is an open access article distributed under the terms and conditions of the Creative Commons Attribution (CC BY) license (http://creativecommons.org/licenses/by/4.0/). 